\title{
NUMERICAL STUDY OF INTERACTIVE MOTION OF DIELECTROPHORETIC PARTICLES ${ }^{*}$
}

\author{
Cuancuan $\mathrm{Xie}^{1)}$, Bo Chen ${ }^{1)}$, Chiu-On $\mathrm{Ng}^{2)}$, Jiankang $\mathrm{Wu}^{3)}$ \\ ${ }^{1}$ (School of Civil Engineering and Mechanics, Huazhong University of Science and Technology, \\ Wuhan, China 430074) \\ ${ }^{2}$ (Department of Mechanical Engineering, The University of Hong Kong, Pokfulam Road, Hong Kong, China) \\ ${ }^{3}$ (Wenhua Institute, Huazhong University of Science and Technology, Wuhan, China 430074)
}

\begin{abstract}
Dielectrophoresis is one of the popular ways to manipulate bio-particles. In this work, numerical simulations of two-dimensional interactive motion of multiple dielectrophoretic particles in an electrolyte subjected to a uniform DC electric field are performed. The dielectrophoretic forces on particles are calculated by an integral of the Maxwell Stress Tensor (MST) on both sides of the particle surfaces. It is shown that behaviors of interactive motion of dielectrophoretic particles are strongly affected by the difference in permittivity between the particles and the electrolyte. Similar particles (all positive or negative electrophoresis) will finally form a chain parallel to the electric field, whereas dissimilar particles (mixed positive and negative electrophoresis) will form a chain that is perpendicular to the electric field. The particle velocity and time behavior of interactive motion are investigated. The key findings are as follows. First, the time required to form a negative particle chain (N-N) is smaller than that for a positive particle chain (P-P) under the same conditions of applied field and permittivity ratio $\left(\varepsilon_{p}: \varepsilon_{m}\right)$. Second, as the ratio $\left(\varepsilon_{p}: \varepsilon_{m}\right)$ increases, the chain-formation time decreases for a P-P particle chain, but increases for an N-N particle chain. Third, the chain-formation time will decrease with increase of the particle size ratio $\left(a_{\max }: a_{\min }\right)$ for a three-particle chain with different particle sizes.
\end{abstract}

Keywords: Dielectrophoresis, particle interactions, Maxwell stress tensor, particle chains.

Supported by National Science Foundation of China, No. 11172111

Corresponding author: Dr. Chen Bo, email: chbo76@hust.edu.cn 


\section{INTRODUCTION}

Dielectrophoresis (DEP) is a phenomenon where a force is exerted on a dielectric particle in a nonuniform electric field [1-3]. The DEP phenomenon was first defined by Pohl [4] based on the equivalent dipole method (EDM). Electrical polarization of finite-size particles can distort the local electric field, resulting in a further nonuniform field around particles. Consequently, an interactive DEP force between particles can be induced even in a uniform electric field. Traditionally the EDM has been widely used for DEP force calculation owing to its simplicity, but it is not accurate in some situations like a high number density or large size of particles $[5,6]$, where the gap between particles is comparable to or smaller than the particle size, and the particles are likely to interact with each other. EDM becomes inapplicable when there is interaction between dielectrophoretic particles. In addition, the Clausius-Mossotti (CM) factor in EDM may not be exactly applicable to non-spherical particles. DEP force calculation based on the Maxwell stress tensor (MST) [6] gives accurate results in all situations in theory, but is cumbersome in implementation. The particles experience hydrodynamic and DEP forces during electrophoresis, while particle motion may distort both the electric and flow fields, which will in turn affect the forces acting on the particles. Fluid-particle-electric field interactions as well as DEP should be fully taken into account in order to accurately predict the electrokinetic particle transport in a microfluidic system. Kang and Li [7] investigated relative motions of a pair of spherical particles in DEP by applying a semi-analytical approximation to the solution of a dielectric force acting on particles and their trajectories. However, their approximate solution is valid only when the initial gap between particles is larger than the particle size. Furthermore, the assumption of a constant Stokes drag is not applicable for DEP particle-particle interaction. House et al. [8] studied the DEP particle-particle interactions for ellipsoidal particles using the boundary element method. The boundary element method, however, can only be used in a linear problem in which a thin electric double layer (EDL) is assumed for the DEP interaction force. Using an arbitrary Lagrangian-Eulerian (ALE) method, Ai and Qian [9-12] performed direct numerical simulations of two-dimensional liquid-solid interaction of a pair of cylindrical particles in an externally applied uniform electric field. In their problem, the flow, electric field and particle motions are solved simultaneously. Later, Kand and Maniyeri [13] performed direct numerical simulations to investigate the DEP interaction of three particles. They employed a finite difference method and a smoothed representation technique to solve for the electric field. Moreover, the particles were considered as completely insulating medium, and therefore the field inside the particles could be ignored. In general, particle polarization in a field is mainly due to a difference in permittivity between the particle and the surrounding electrolyte. The particle permittivity should therefore be taken into account for a complete DEP study. Recently, Hossan and Dillon [14] investigated the DEP motions of multiple particles using a hybrid immersed interface-immersed boundary method with an aim to examine DEP particle interaction including effect of permittivity difference between the particles and the electrolyte. To the best knowledge of the authors, DEP particle interaction of multi-particles with different sizes has not been studied in the existing literature. The main objective of this work is to explore dynamic 
behavior of interactive motion of multiple particles with different sizes in an applied uniform electric field via numerical solutions based on the full Navier-Stokes equation and an Arbitrary Lagrangian-Eulerian algorithm for liquid-solid interaction.

\section{VALIDATION OF NUMERICAL ACCURACY OF DIELECTROPHORETIC FORCE BY MAXWELL STRESS TENSOR (MST)}

To verify the numerical accuracy of MST in calculating electrophoretic force, a comparison is made between the dipole approximation method and the MST method when they are applied to a numerical example as detailed in Refs. [15,16]. A cylindrical particle with permittivity $\varepsilon_{p}$ is immersed in an electrolyte with permittivity $\varepsilon_{f}$ Based on the equivalent dipole method, the DEP force acting on the particle can be written as follows [15]:

$$
\begin{aligned}
& f_{x}=2 C\left[\frac{\partial \phi}{\partial x} \frac{\partial^{2} \phi}{\partial x^{2}}+\frac{\partial \phi}{\partial y} \frac{\partial^{2} \phi}{\partial x \partial y}\right] \\
& f_{y}=2 C\left[\frac{\partial \phi}{\partial x} \frac{\partial^{2} \phi}{\partial x \partial y}+\frac{\partial \phi}{\partial y} \frac{\partial^{2} \phi}{\partial y^{2}}\right]
\end{aligned}
$$

where $\phi$ is the applied electric potential without particles, and the constant $C$ is given by

$$
C=\pi \varepsilon_{f} \frac{\varepsilon_{p}-\varepsilon_{f}}{\varepsilon_{p}+\varepsilon_{f}} a^{2}
$$

The subscripts $f$ and $p$ denote the fluid and the particle, respectively, and $a$ is the particle radius. Beause the permittivity and electrical field are discontinuous across the particle-fluid interface, one needs to calculate MST on both sides of the particle surface [15,16]. The Maxwell stress tensors read as follows:

$$
\boldsymbol{T}_{p}^{E}=\varepsilon_{p}\left[\boldsymbol{E}_{p} \boldsymbol{E}_{p}-\frac{1}{2}\left(\boldsymbol{E}_{p} \cdot \boldsymbol{E}_{p}\right) \boldsymbol{I}\right]
$$

for particle side of surface

$$
\boldsymbol{T}_{f}^{E}=\varepsilon_{f}\left[\boldsymbol{E}_{f} \boldsymbol{E}_{f}-\frac{1}{2}\left(\boldsymbol{E}_{f} \cdot \boldsymbol{E}_{f}\right) \boldsymbol{I}\right]
$$

for fluid side of surface 
where $I$ denotes the second-order unit tensor, and $\boldsymbol{E}_{f}$ and $\boldsymbol{E}_{p}$ are the electric field in the fluid and in the particle, respectively. The electrophoretic force of particle is obtained by an integral of MST over the particle surface:

$$
\mathbf{F}_{\mathrm{dep}}=\oint\left(\mathbf{T}_{p}^{E}-\mathbf{T}_{f}^{E}\right) \cdot \mathbf{n} d s
$$

where $\mathbf{n}$ is the unit normal vector on the particle surface pointing to the fluid. The numerical example used for validation and the pertained parameters are given in Ref. [15]. A particle is located in an electric field, as shown in Fig. 1. For simplicity, the numerical details are not presented here.

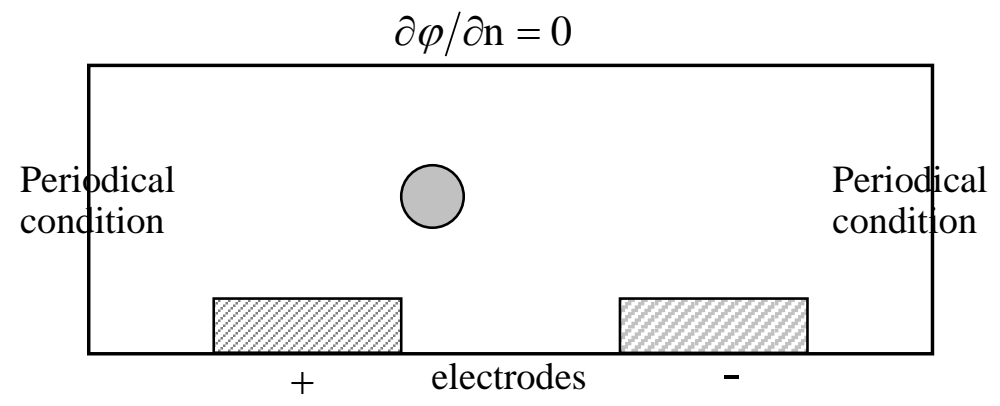

Fig.1 Computational domain and boundary conditions of the numerical example for validation.

A comparison of the electrophoretic force on a cylindrical particle as calculated by the MST and the dipole approximation is shown in Fig. 2.
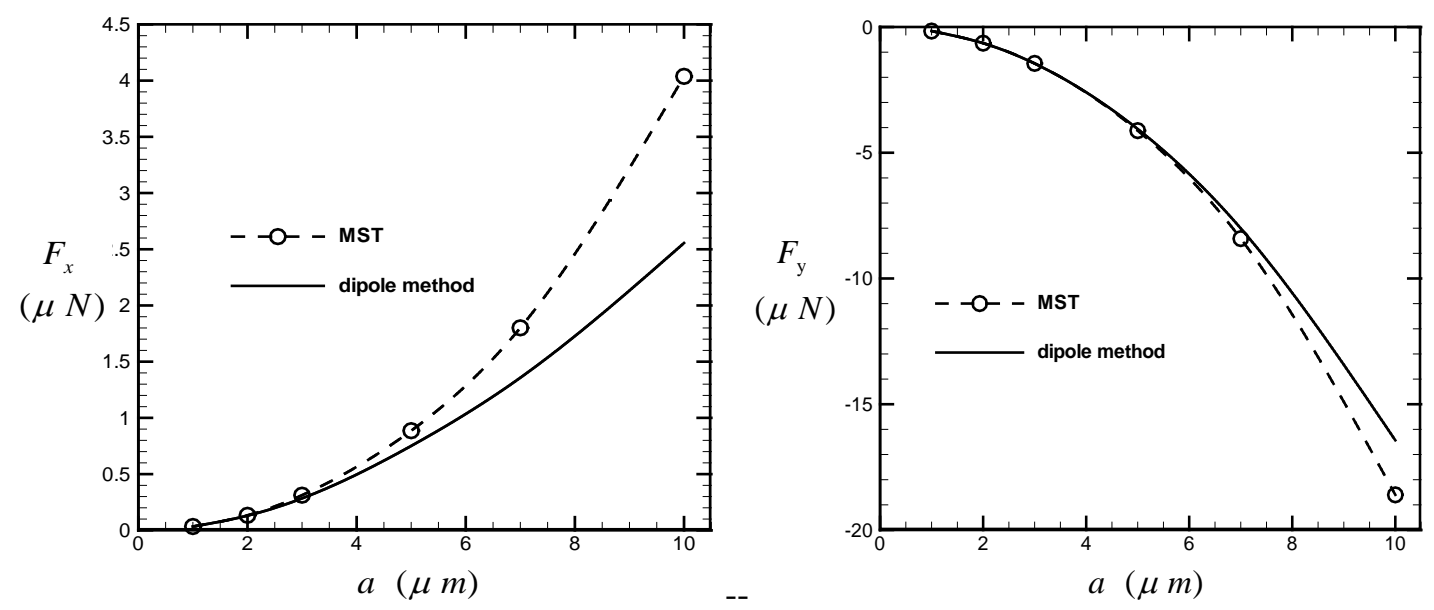

Fig. 2 Comparison of DEP force on a circular cylinder particle calculated using dipole approximation and MST methods, as a function of particle radius. 
It can be seen that dipole approximation method and MST method give almost the same DEP force for a small particle, $a \leq 4 \mu \mathrm{m}$ in the present example. Deviation of results generated by the two methods increases with increase of the particle radius. It has been known that the dipole approximation is based on the point-dipole assumption, which is applicable only for a single small particle, while the MST is theoretically exact in general.

\section{GOVERNING EQUATIONS AND BOUNDARY CONDITIONS FOR PARTICLE INTERACTION IN AN ELECTRIC FIELD}

Consider a pair of similar particles suspended in a viscous incompressible fluid in an $L \times L$ square domain (ABCD). A uniform electric field $\mathbf{E}$ is applied, as shown in Fig. 3, where $a$ is the particle radius, $R$ is the distance between the two particle centers, $\theta$ is the initial directional angle between the connecting line of the two particles and the $x$-axis, $\Omega 1, \Omega 2$ and $\Omega 3$ denote the fluid domain and the two particle domains, respectively, and $\Gamma_{1}, \Gamma_{2}$ denote the surfaces of the two particles.

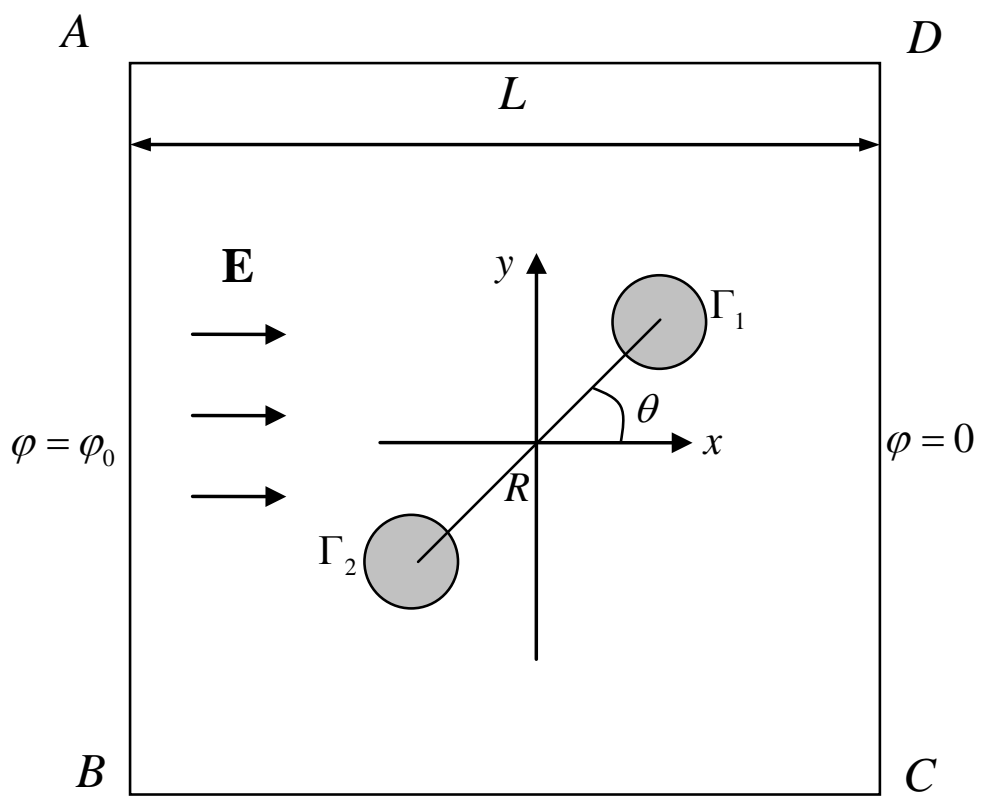

Fig. 3 A pair of similar particles suspended in electrolyte subjected to an externally applied electric field $\mathbf{E}$.

Let us use the particle radius $a$ as the length scale, the electric potential $\varphi_{0}$ as the potential scale, $U_{0}=\varepsilon_{f} \varphi_{0}^{2} /(\eta a)$ as the velocity scale, and $\eta U_{0} / a$ as the pressure scale. Then, the normalized electric potential is governed by the Laplace equation: 


$$
\nabla^{2} \phi=0
$$

The electric potentials on $\mathrm{AB}$ and $\mathrm{CD}$ are specified as $\phi=1$ and $\phi=0$, respectively. The other boundaries are insulated walls: $\boldsymbol{n} \cdot \nabla \phi=0, \quad \varphi_{p}=\varphi_{f}, \quad \varepsilon_{p} \frac{\partial \varphi_{p}}{\partial n}=\varepsilon_{f} \frac{\partial \varphi_{f}}{\partial n}$ on the particle surfaces $\Gamma_{1}, \Gamma_{2}$. The Reynolds number of fluid flow in dielectrophoresis is typically so low that the fluid inertia can be neglected. The equations governing mass and momentum conservation of the fluid are as follows:

$$
\begin{gathered}
\nabla \cdot \boldsymbol{u}=0 \\
\operatorname{Re} \frac{\partial \boldsymbol{u}}{\partial t}-\nabla^{2} \boldsymbol{u}+\nabla p=0
\end{gathered}
$$

where $\operatorname{Re}=\rho a U_{0} / \eta$, in which $\rho, \eta$ are the fluid density and dynamic viscosity. The fluid velocity on the particle surface is related to the translational and rotational velocities of the particle, expressed as [12]:

$$
\boldsymbol{u}_{i}=\boldsymbol{U}_{p i}+\boldsymbol{\omega}_{p i} \times\left(\boldsymbol{x}_{s i}-\boldsymbol{x}_{p i}\right) \quad i=1,2 \text { on } \Gamma_{1}, \Gamma_{2}
$$

where $\mathbf{U}_{p i}$ and $\boldsymbol{\omega}_{p i}$ are the translational velocity and rotational velocity of the ith particle, and $\boldsymbol{x}_{s i}$ and $\boldsymbol{x}_{p i}$ are the position vector of the surface, and of the center of the ith particle, respectively. A symmetry condition is imposed on the boundaries $\mathrm{BC}$ and $\mathrm{AD}$, and zero pressure is specified on the boundaries $\mathrm{AB}$ and $\mathrm{CD}$. The particles are assumed to be far away from the boundaries. The force and torque being normalized by $\eta U_{0}$ and $a \eta U_{0}$, respectively, the translation and rotation of the ith particle are governed by [12]:

$$
\begin{gathered}
m_{p i} \frac{d \boldsymbol{U}_{p i}}{d t}=\boldsymbol{F}_{H i}+\boldsymbol{F}_{E i} \\
I_{p i} \frac{d \boldsymbol{\omega}_{p i}}{d t}=\oint\left(\boldsymbol{x}_{s i}-\boldsymbol{x}_{p i}\right) \times\left[\left(\boldsymbol{T}_{H}+\boldsymbol{T}_{E}\right) \cdot \boldsymbol{n}\right] d s_{i}
\end{gathered}
$$

where the mass $m_{p i}$ and the moment of inertia $I_{p i}$ of the ith particle are normalized by $a \eta U_{0}$ and $a^{3} \eta U_{0}$, respectively. $\mathbf{F}_{H i}$ and $\mathbf{F}_{E i}$ are the hydrodynamic force and the DEP force, which are obtained by integrating the hydrodynamic stress tensor $\left(\mathbf{T}_{H}\right)$ and the MST $\left(\mathbf{T}_{E}\right)$ over the surface of the ith particle:

$$
F_{H i}=\oint \boldsymbol{T}_{H} \cdot \boldsymbol{n} d s_{i}=\oint\left[-p \boldsymbol{I}+\left(\nabla \boldsymbol{u}+\nabla \mathbf{u}^{T}\right)\right] \cdot \boldsymbol{n} d s_{i}
$$




$$
\boldsymbol{F}_{E i}=\oint\left[\frac{\varepsilon_{p} E_{p} E_{p}}{\varepsilon_{f}}-\frac{\varepsilon_{p}\left(E_{p} \cdot E_{p}\right) \boldsymbol{I}}{2 \varepsilon_{f}}-E_{f} E_{f}+\frac{1}{2}\left(E_{f} \cdot E_{f}\right) \boldsymbol{I}\right] \cdot \boldsymbol{n} d s_{i}
$$

\section{RESULTS AND DISCUSSION}

\subsection{Interactive motions of a pair of similar particles}

A particle with permittivity ratio $\varepsilon_{p} / \varepsilon_{f}>1$ is defined as a positive dielectrophoretic particle, abbreviated as a P particle in this paper. A particle with $\varepsilon_{p} / \varepsilon_{f}<1$ is defined as a negative dielectrophoretic particle, or an $\mathrm{N}$ particle. Particles are called similar when they are either all $\mathrm{P}$ particles (P-P), or all N particles (N-N). Particles are called dissimilar when there are $\mathrm{P}$ and $\mathrm{N}$ particles in the group (P-N). A pair of identical particles initially located at $R=3 a$ and $\theta=85^{\circ}$ will turn around clockwise, finally forming a particle chain aligned with the electric field, as shown in Fig. 4, where the colors are contours of the electric field and the lines are flow streamlines.

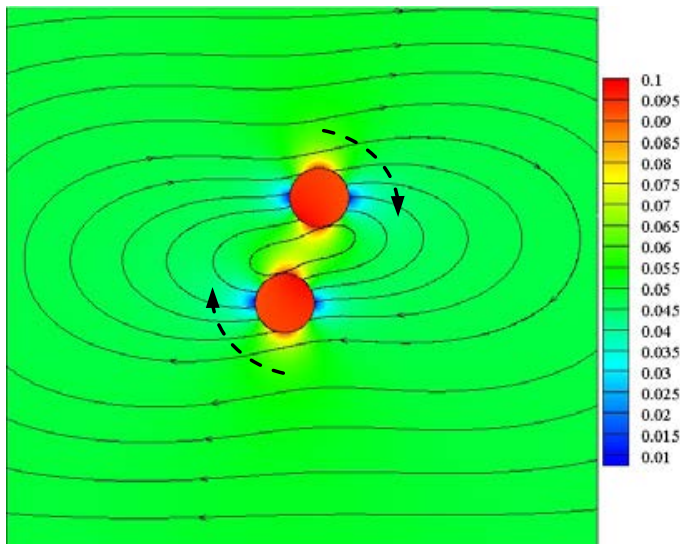

Fig. 4a Initial configuration of a pair of $\mathrm{N}-\mathrm{N}$ particles.

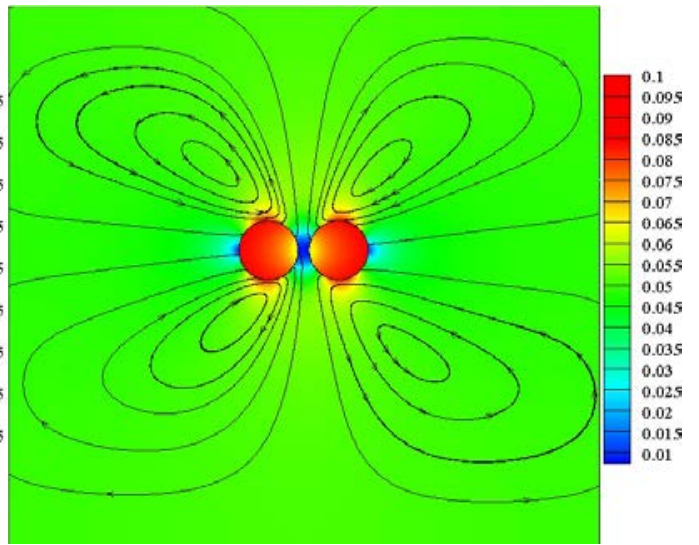

Fig. 4b Final alignment of a particle chain of a pair of $\mathrm{N}-\mathrm{N}$ particles $\varepsilon_{p} / \varepsilon_{f}=0.1$. 


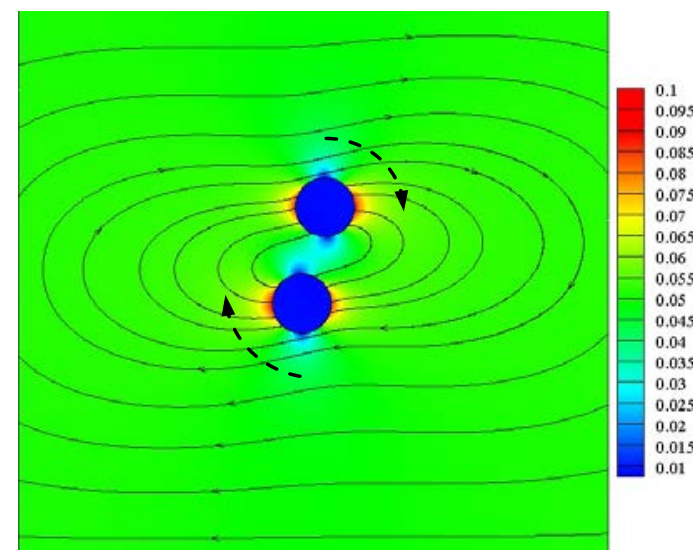

Fig. 4c Initial configuration of a pair of P-P particles.

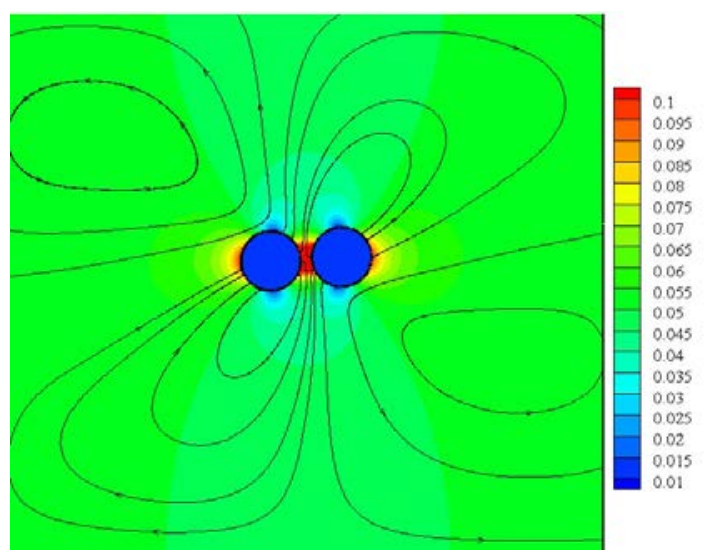

Fig.4d Final alignment of a particle chain of a pair of P-P particles $\varepsilon_{p} / \varepsilon_{f}=10$.

It can be seen from Figs. 4(c, d) that the particles will attract each other. The N-N particles tend to move toward a lower field, while the P-P particles tend to move toward a higher field. The motion trajectories of a pair of identical particles are shown in Fig. 5.

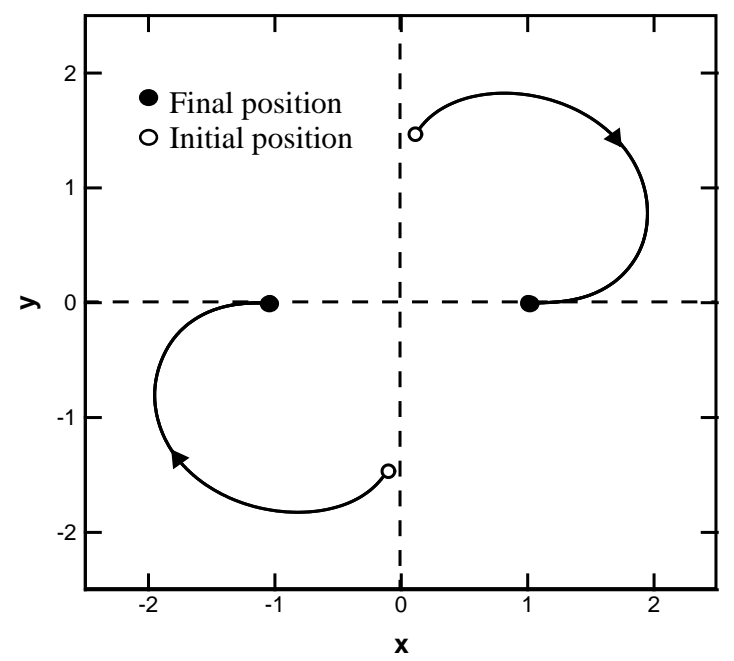

Fig. 5 Trajectories of interactive motion of a pair of identical N-N particles initially located at $R=3 \mu \mathrm{m}$ and $\theta=85^{\circ}$.

In the region of $45^{\circ}<\theta<90^{\circ}$, the particles will experience a repulsive force, by which they move outward and revolve in the clockwise direction. In the region of $\theta<45^{\circ}$, the particles will on the contrary experience an attractive force, by which they move inward and move clockwise. Finally, the two particles will get close to each other forming a chain that is aligned with the electric field, as is shown in Figs. 4(c, d). These 
behaviors are consistent with those reported previously in Refs. [12-14]. Motion trajectories of the upper particle of the similar particle chain are shown in Fig. 6 for different values of the permittivity ratio $\left(\varepsilon_{p} / \varepsilon_{m}\right)$. It can be seen that the trajectory length of P-P particles $\left(\varepsilon_{p} / \varepsilon_{m}>1\right)$ is shorter than that of N-N particles $\left(\varepsilon_{p} / \varepsilon_{m}<1\right)$ for the same permittivity ratio. Moreover, the trajectory length increases with a decreasing ratio of $\varepsilon_{p} / \varepsilon_{m}$. The trajectory behavior of the lower particle is the same as that of the upper one.

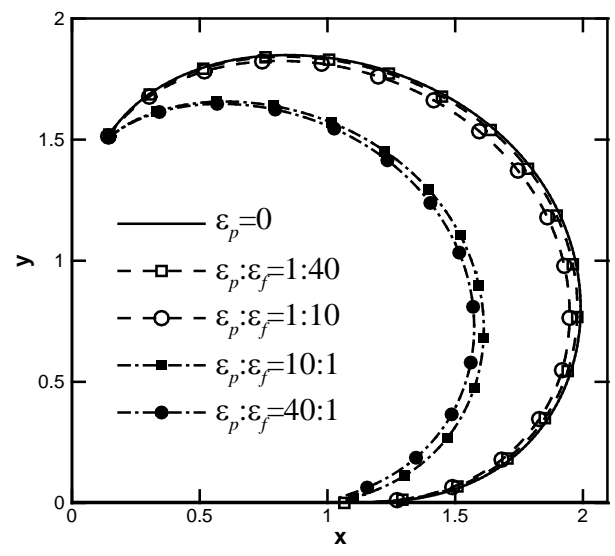

Fig. 6. Motion trajectories of the upper particle in a similar particle chain for different permittivity ratio $\varepsilon_{p} / \varepsilon_{m}$.

The time variations of the velocity of the lower particle in a pair of similar particles in the chain-forming process are shown in Figs. 7-10.

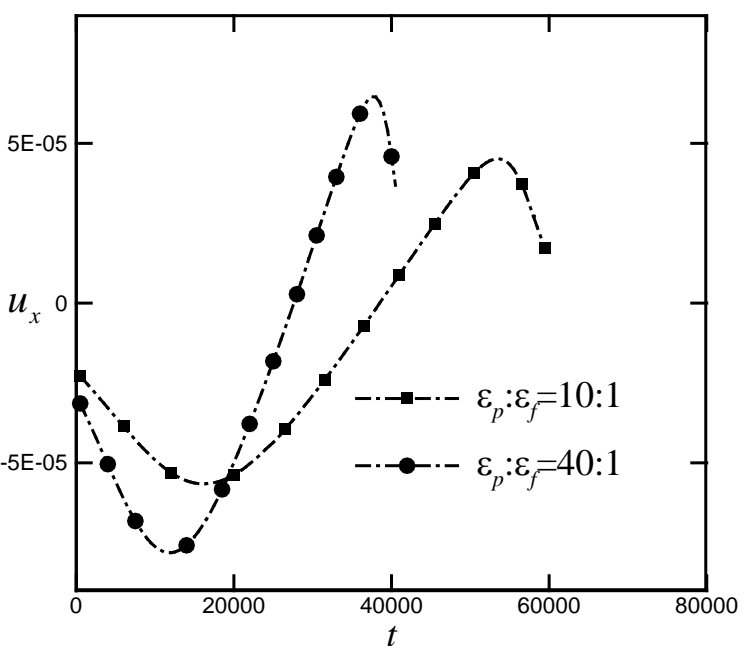

Fig. 7 The time variations of the $x$-velocity of the lower particle in a pair of P-P particles.

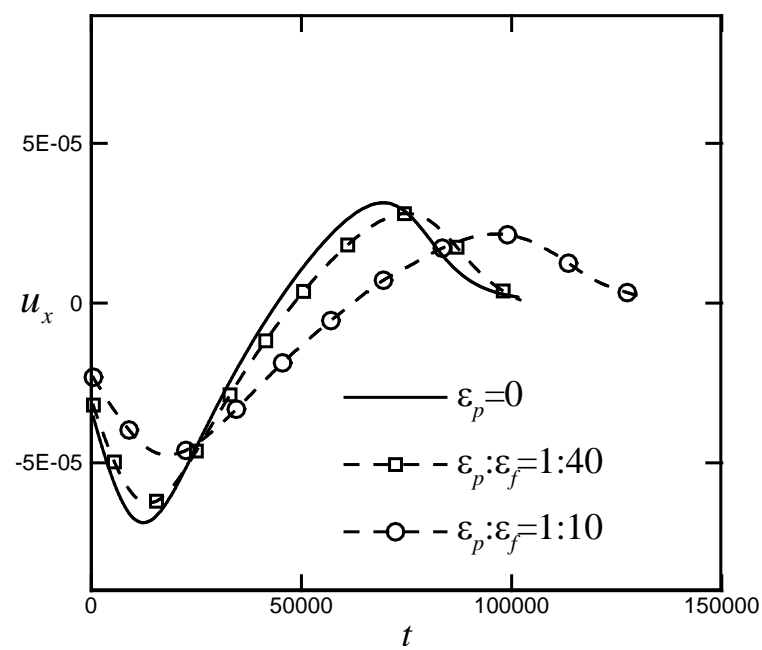

Fig. 8 The time variations of the $x$-velocity of the lower particle in a pair of $\mathrm{N}-\mathrm{N}$ particles. 


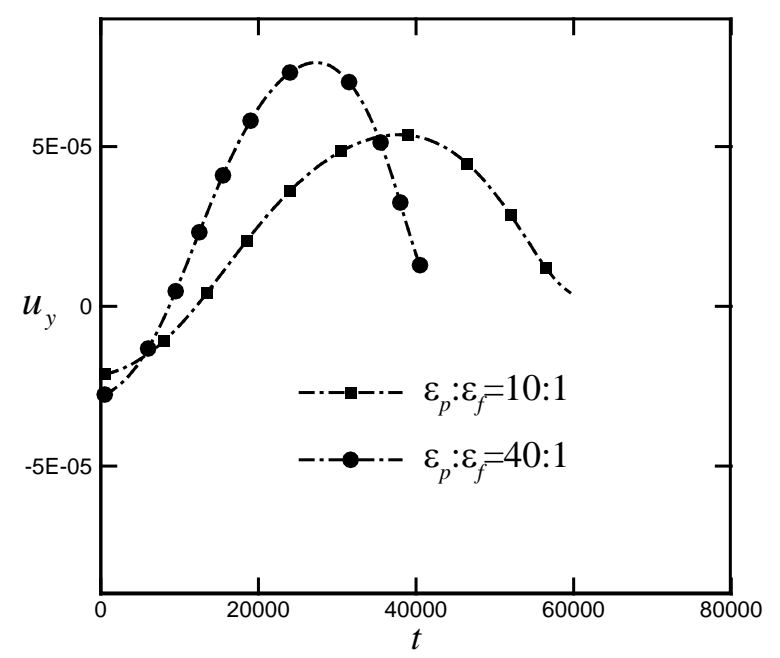

Fig. 9 The time variations of the $y$-velocity of the lower particle in a pair of P-P particles.

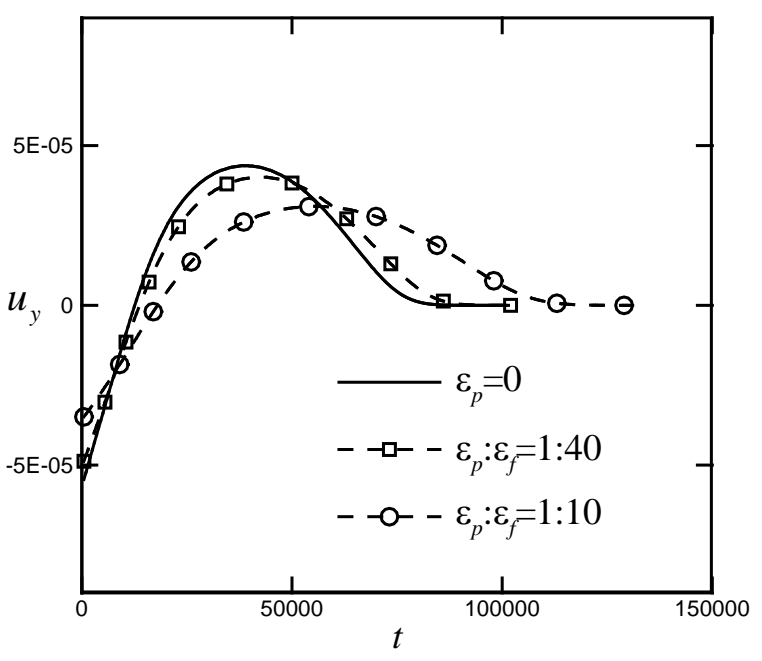

Fig. 10 The time variations of the $y$ velocity of lower particle in a pair of N-N particles.

When the particle velocity decreases to zero, a stable particle chain is formed. It can be seen from Fig. 9 that the traveling time of P-P particles in the course of chain formation increases with decrease of the ratio $\varepsilon_{p} / \varepsilon_{m}$. The decrease of the ratio $\varepsilon_{p} / \varepsilon_{m}$ implies a decrease of $\left(\Delta \varepsilon=\varepsilon_{p}-\varepsilon_{f}\right)$, and hence as the DEP force and the particle velocity decreases, the traveling time for chain formation will increase. To the contrary, the traveling time for chain formation of $\mathrm{N}-\mathrm{N}$ particles decreases as the ratio $\varepsilon_{p} / \varepsilon_{m}$ decreases, see Fig. (10). For N-N particles, the decrease of ratio $\varepsilon_{p} / \varepsilon_{m}$ leads to an increase of $\left(\Delta \varepsilon=\varepsilon_{p}-\varepsilon_{f}\right.$ ), and therefore as the DEP force and the particle velocity increase, the chain formation time will decrease.

\subsection{Interactions of a pair of dissimilar particles}

For a pair of P-N particles initially located at $R=3 a, \theta=10^{\circ}$ with $\varepsilon_{1 p}: \varepsilon_{f}: \varepsilon_{2 p}=1: 10: 100$, the particles will turn around counterclockwise, as is shown in Fig. (8). 


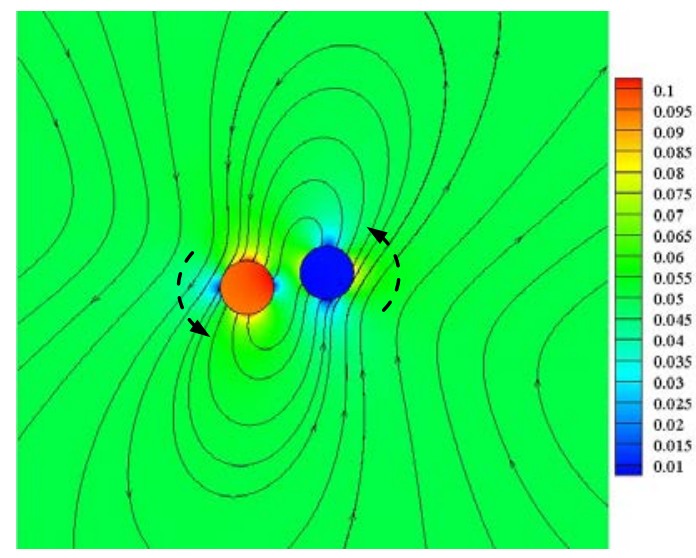

Fig. 8a Initial configuration of a pair of N-P particles with $\varepsilon_{1 p}: \varepsilon_{f}: \varepsilon_{2 p}=1: 10: 100$.

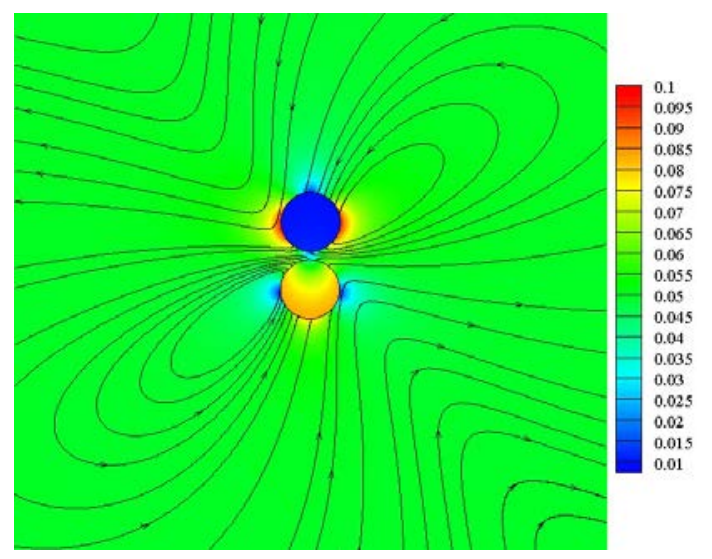

Fig. 8b Final alignment of a particle chain of a pair of N-P particles with $\varepsilon_{1 p}: \varepsilon_{f}: \varepsilon_{2 p}=1: 10: 100$.

Fig. 8(b) shows that a stable dissimilar-particle-chain is finally formed, aligned in a direction perpendicular to the electric field. The P particle (deep blue) tends to move toward a higher field (green), while the N particle (yellow) tends to move toward a lower field (green). The motion trajectories of a pair of dissimilar particles are shown in Fig. 9.

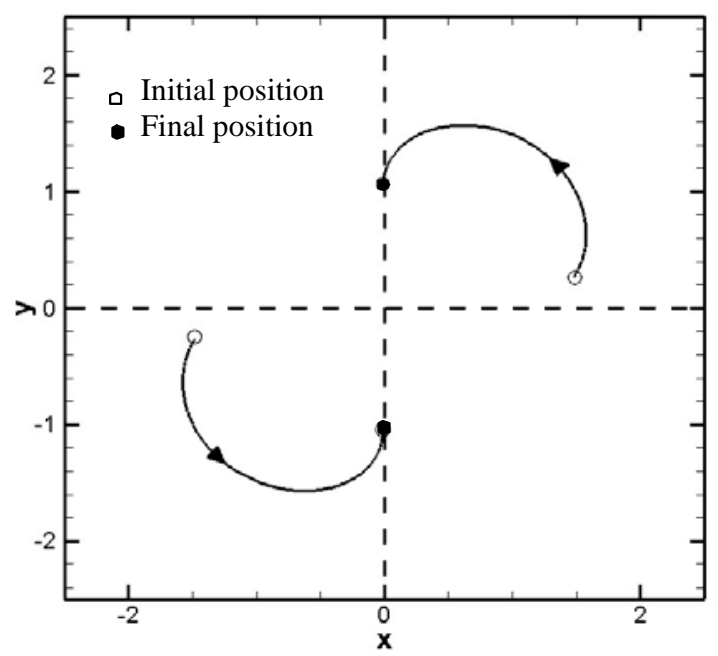

Fig. 9. Trajectories of a pair of dissimilar particles initially located at $R=3 a, \theta=10^{\circ}$.

It can be seen that in the region $0^{\circ}<\theta<45^{\circ}$, the particles experience a repulsive force by which they move outward, and turn around counterclockwise. In the region of $\theta<45^{\circ}$, the particles experience an attractive force, by which they move inward while revolving. Finally, an N-P particle chain is formed. 
4.3. Three identical particles interactions and relative motions

Consider three identical particles in a uniform electrical field, as is shown in Fig. 10.

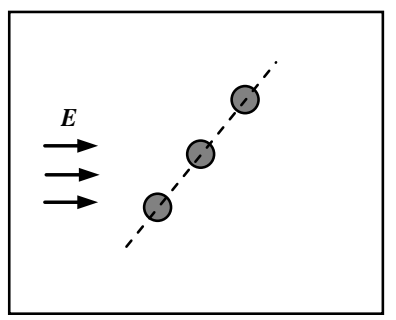

Fig. 10 Three particles suspended in a square domain of electrolyte in a uniform electric field.

The initial configuration of three $\mathrm{N}$ particles and the final particle chain are shown in Figs. 11-12, where $\varepsilon_{p}: \varepsilon_{f}=1: 10$

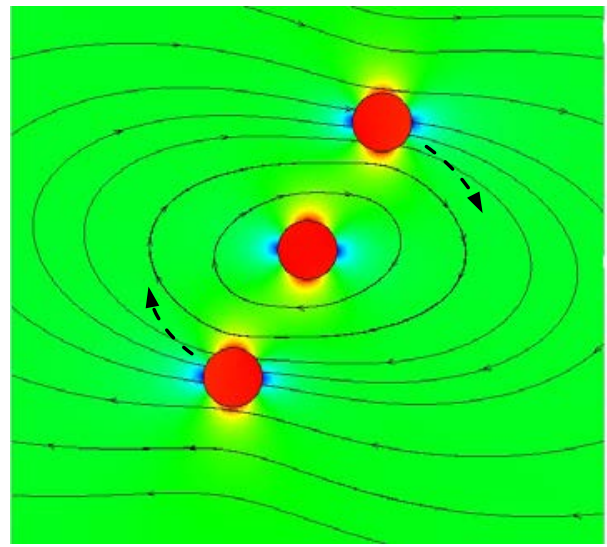

Fig. 11 Three $N$ particles initially located at $R=5 a, \theta=60^{\circ}$ in a uniform electrical field.

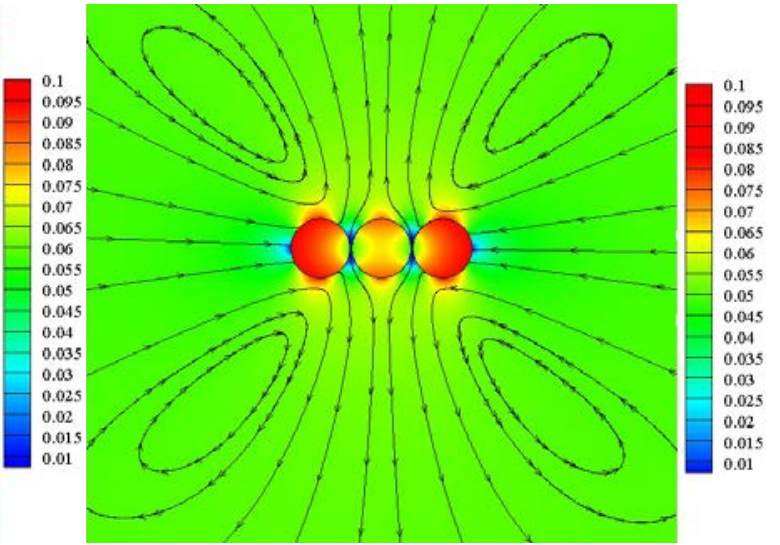

Fig. 12 Final alignmnet of a particle chain of three $\mathrm{N}$ particle in a uniform electrical field.

The initial configuration of three dissimilar particles and the final particle chain are shown in Figs. 13-14, where $\varepsilon_{p 1}: \varepsilon_{p 2}: \varepsilon_{f}: \varepsilon_{p 3}=1: 1: 10: 100$. 


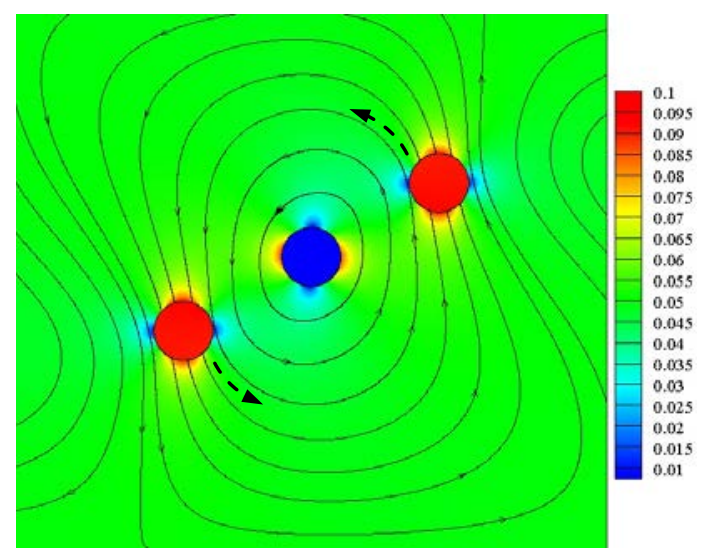

Fig.13 Three dissimilar particles initially located at $R=5 a, \theta=30^{\circ}$ in a uniform electrical field.

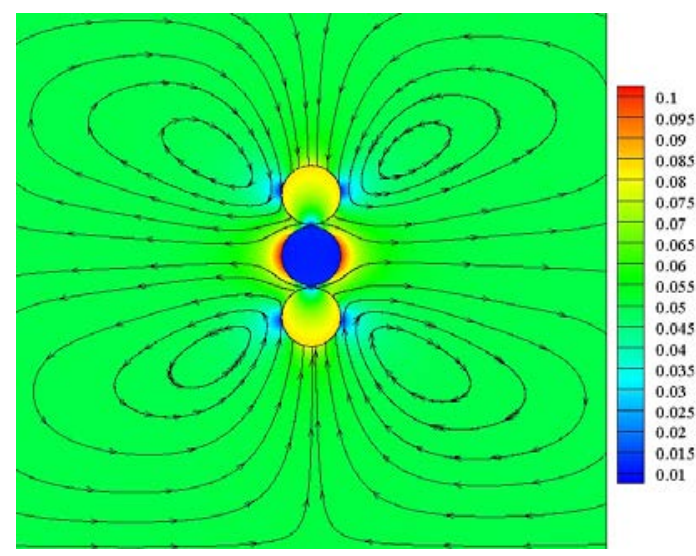

Fig.14 Final alignment of a particle chain of three dissimilar particles in a uniform electrical field.

The trajectories of three particles are shown in Figs. 15-16.

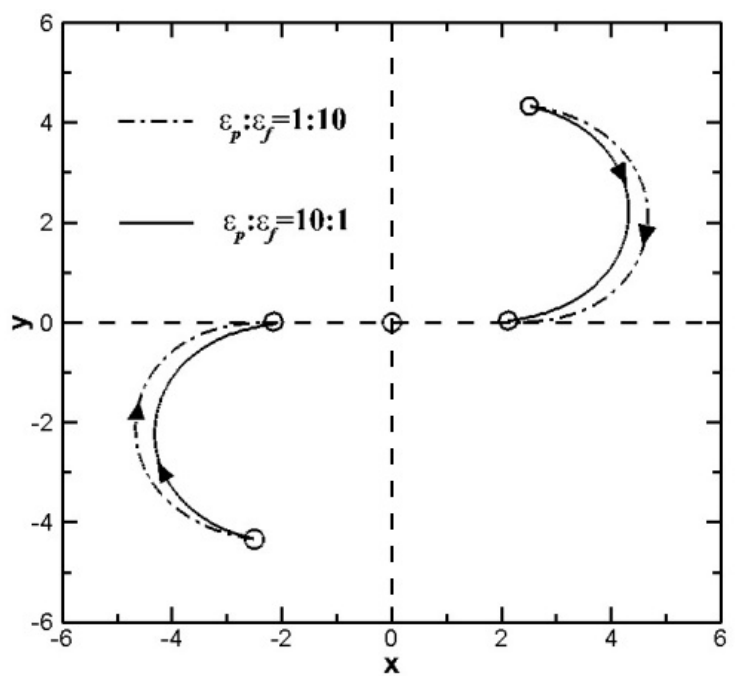

Fig. 15 Motion trajectories of three similar particles.

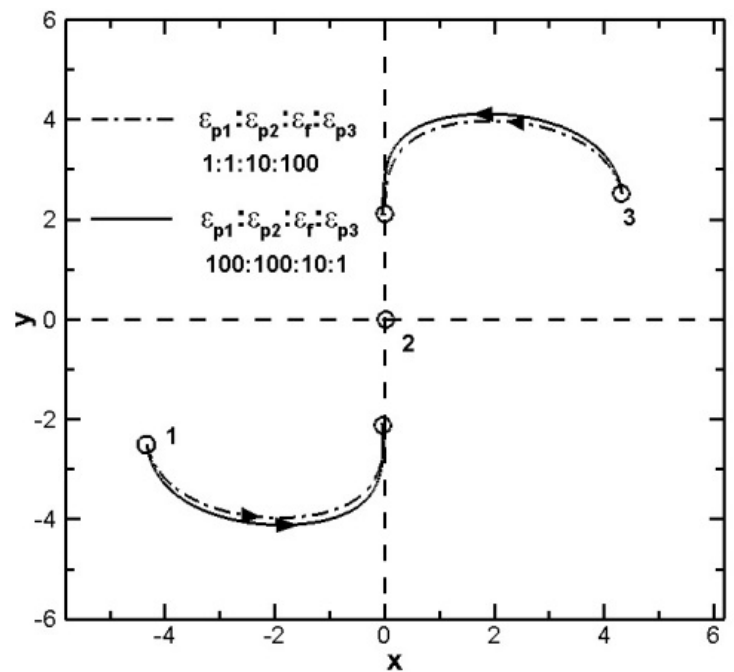

Fig. 16 Motion trajectories of three dissimilar particles.

The initial configuration and the final particle chain of three similar particles with different sizes are shown in

Figs. 17-18, where $\varepsilon_{p} / \varepsilon_{f}=0.1$, and $a_{\max }: a_{\min }=2$. 


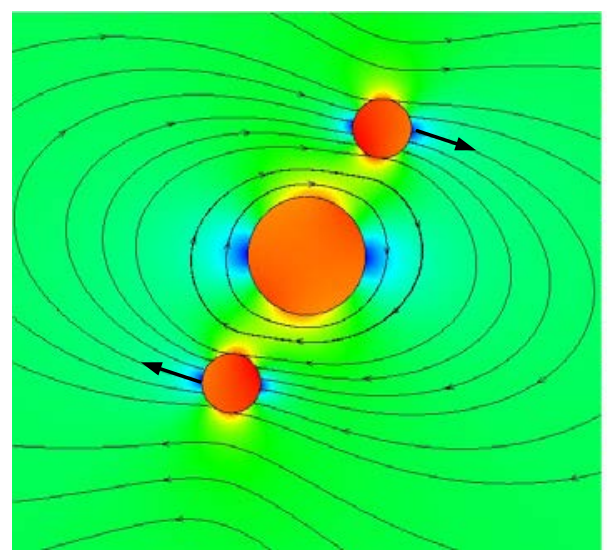

Fig.17 Three $\mathrm{N}$ particles of different sizes initially located at $R=5 a, \theta=60^{\circ}$ in a uniform electrical field.

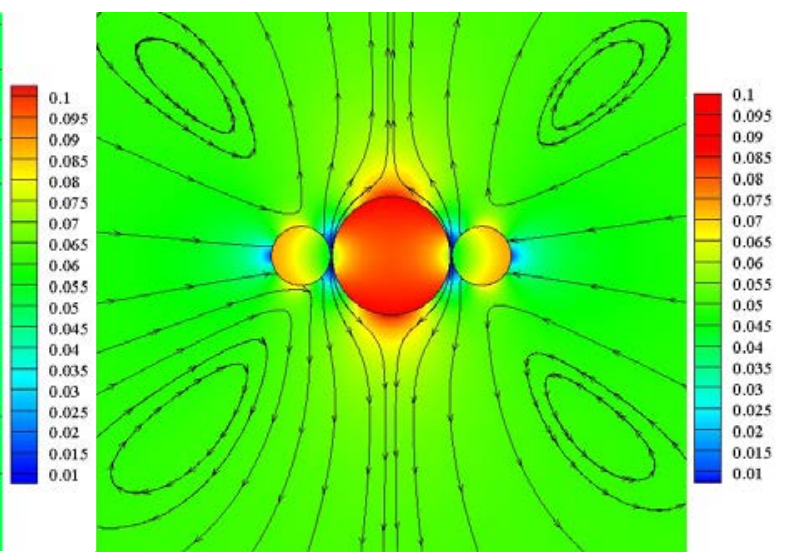

Fig.18 Final alignment of a particle chain of three $\mathrm{N}$ particles of different sizes in a uniform electrical field.

The initial configuration and the final particle chain of three dissimilar particles with different sizes are shown in Figs. 19-20, where $\varepsilon_{p 1}: \varepsilon_{p 2}: \varepsilon_{f}: \varepsilon_{p 3}=1: 1: 10: 100$, and $a_{\max }: a_{\min }=2$.
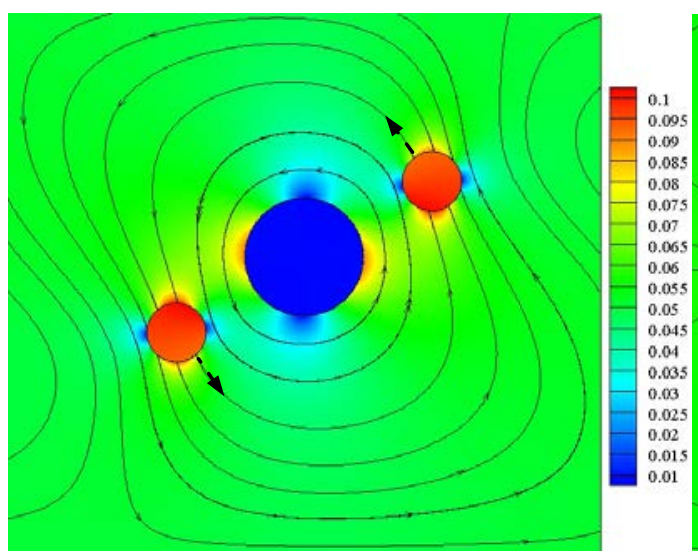

Fig. 19 Three dissimilar particles of different sizes initially located at $R=5 a, \theta=30^{\circ}$ in a uniform electrical field.

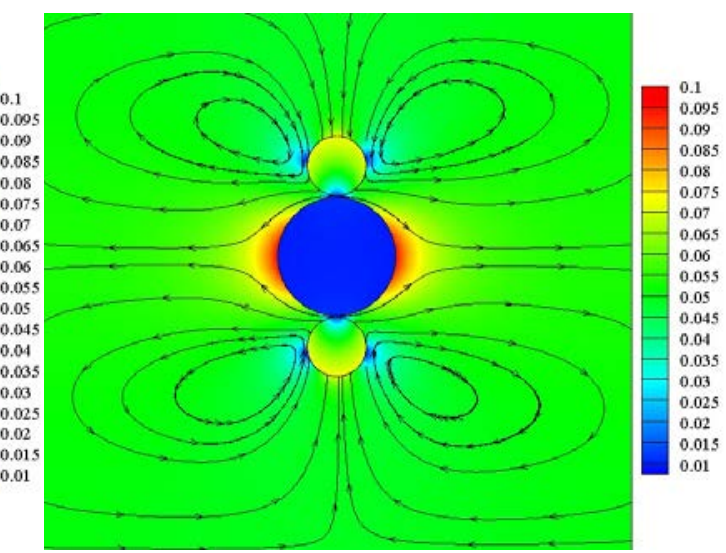

Fig. 20 Final alignment of a particle chain of three dissimilar particles of different sizes in a uniform electrical fields 
The variations with time of the velocity of the lower particle in a group of three similar particles with different sizes are shown in Fig. 21.

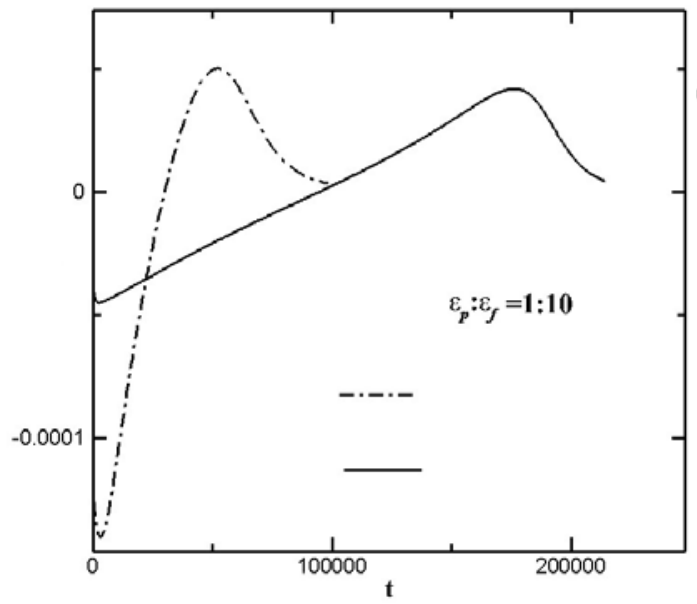

(a)

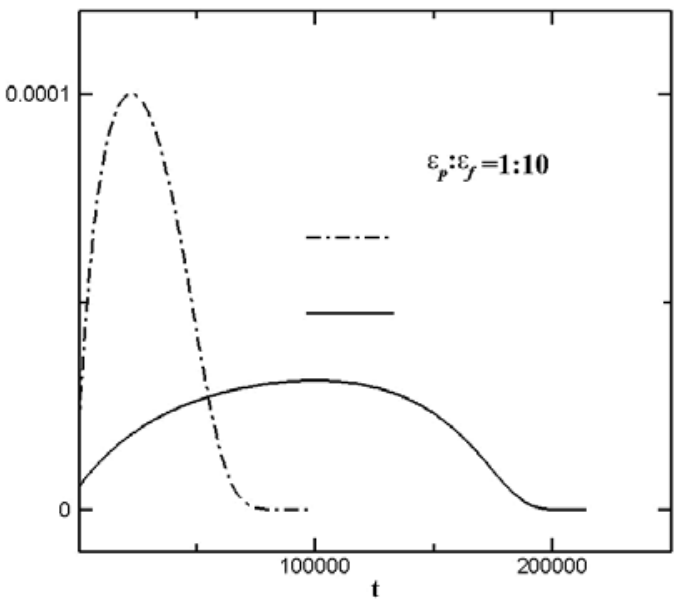

(b)

Fig. 21 Time variations of velocity of the lower particle in a group of three $\mathrm{N}$ particles with different sizes ( $\varepsilon_{p} / \varepsilon_{f}=1: 10$ ): (a) X-velocity, (b) Y-velocity.

It can be seen that the velocities of the particles with a size ratio of $a_{\max } / a_{\min }=2$ are larger than those of particles with a size ratio of $a_{\max } / a_{\min }=1$, and the chain formation time for the particles of $a_{\max } / a_{\min }=2$ is much shorter than that for the particles of $a_{\max } / a_{\min }=1$. Similar to the two-particle cases, a similar threeparticle chain is aligned with the electric field, while a dissimilar three-particle chain is aligned perpendicular to the electric field. Also, as the particle size ratio increases, the particle velocity increases, while the particle trajectories and the chain formation time decreases. The non-uniformity of local electric field increases as the particle size ratio increases.

\section{CONCLUSIONS}

Effects on DEP particle interaction motion in a uniform electric field due to difference in permittivity between particle and fluid and the particle size ratio are numerically studied. Similar particle chains are ultimately aligned with the electric field, while dissimilar particle chains are aligned perpendicular to the electric field. The time required to form a negative particle chain $(\mathrm{N}-\mathrm{N})$ is smaller than that for a positive particle chain (P-P) for the same applied field and permittivity ratio $\left(\varepsilon_{p}: \varepsilon_{m}\right)$. As the ratio $\left(\varepsilon_{p}: \varepsilon_{m}\right)$ increases, the chainformation time decreases for P-P similar particle chains, but increases for N-N similar particle chains. The chain-formation time will decrease with an increasing particle size ratio $\left(a_{\max }: a_{\min }\right)$ for a three-particle chain. 


\section{References}

[1] T. B. Jones, Electromechanics of particles, Cambridge University Press, Cambridge, UK (1995).

[2] H. Morgan and N. G. Green, AC electrokinetics: colloids and nanoparticles, Research Studies Press, Philadelphia, USA (2002).

[3] Y. Kang and D. Li, Electrokinetic motion of particles and cells in microchannels, Microfluidics and Nanofluidics, 6 (2009) 431-460.

[4] H.A. Plhl, Dielectrophoresis, Cambridge University Press, Cambridge, England (1978).

[5] N. Aubry, P. Singh, Control of electrostatic particle-particle interactions in dielectrophoresis, Europhys. Lett. 74 (2006), 623-629.

[6] S. Kumar, Peter J. Hesketh, Interpretatio of ac dielectrophoretic behavior of tin oxide nanobelts using Maxwell stress tensor approach modeling, Sensors and Actuators B, 161 (2012) 1198-1208.

[7] K. H. Kang and D. Li, Dielectric force and relative motion between two spherical particles in electrophoresis, Langmuir, 22 (2006) 1602-1608.

[8] D.L. House, H.X. Luo, S.Y. Chang, Numerical study on dielectrophoretic chaining of two ellipsoidal particles, J. Colloid Interface Sci. 374 (2012) 141-149.

[9] Y. Ai, S. W. Joo, Y. Jiang, X. Xuan, and S. Qian, Transient electrophoretic motion of a charged particle through a converging-diverging microchannel: Effect of direct current-dielectrophoretic force, Electrophoresis, 30 (2009) 2499-2506.

[10] Y. Ai, A. Beskok, D. T. Gauthier, S. W. Joo, and S. Qian, DC electrokinetic transport of cylindrical cells in straight microchannels, Biomicrofluidics, 3 (2009) 044110.

[11] Y. Ai, S. Park, J. Zhu, X. Xuan, A. Beskok, and S. Qian, DC electrokinetic particle transport in an Lshaped microchannel, Langmuir, 26 (4) (2010) 2937-2944.

[12] Y. Ai, and S. Qian, DC dielectrophoretic particle-particle interactions and their relative motions, Journal of Colloid and Interface Science, 346 (2010) 448-454.

[13] Sangmo Kang, and Ranjith Maniyeri, Dielectrophoretic motions of multiple particles and their analogy with the magnetophoretic counterparts, Journal of Mechanical Science and Technology, 26(11) (2012) 3503-3513.

[14] Mohammad Robiul Hossan, Robert Dillon and Ajit K. Roy, Prashanta Dutta, Modeling and simulation of dielectrophoretic particle-particle interactions and assembly, Journal of Colloid and Interface Science, 394 (2013) 619-629. 
[15] Eugeniusz Kurgan, Stress Calculation in Two-Dimensional DC Dielectrophoresis, Electrical Review, 87(12b) (2011) 0033-2097.

[16] Eugeniusz Kurgan, Comparison of different force calculation methods in DC dielectrophoresis, Electrical Review, 88(8) (2011) 0033-2097.

[17] C.H. Kua, Y.C. Lam, I. Rodriguez, C. Yang, K. Youcef-Toumi, Cell Motion Model for Moving Dielectrophoresis, Anal. Chem., 80 (14) (2008) 5454-5461. 